\title{
Radiation from accelerated particles at strong coupling
}

\author{
Daniel Fernandez-Fraile* \\ Institut für Theoretische Physik, Johann Wolfgang Goethe-Universität, Max-von-Laue-Str. 1, \\ 60438 Frankfurt am Main, Germany \\ E-mail: danfereth.physik.uni-frankfurt.de
}

\begin{abstract}
We report on our work in progress studying the power and angular distribution of the radiation emitted by an accelerated particle at strong coupling. For that purpose, we use the framework of the AdS/CFT conjecture. The acceleration in our case is caused by an external magnetic field which couples at every loop order. Consequently, any state (charged or neutral under the interaction with the magnetic field) is accelerated. For intense-enough magnetic fields, there is an effective dimensional reduction from $3+1$ to $1+1$ dimensions, which qualitatively modifies the pattern of radiation with respect to results obtained by previous studies at weak and strong coupling.
\end{abstract}

36th International Conference on High Energy Physics,

July 4-11, 2012

Melbourne, Australia

${ }^{*}$ Speaker. 


\section{Introduction}

The AdS/CFT conjecture [1] has opened the possibility to carrying out analytical calculations at strong coupling for many observables of some non-abelian gauge theories in $3+1$ dimensions. Before the conjecture was put forward by Maldacena, some of these computations were only conceivable by means of lattice techniques. It essentially establishes the duality (i.e., equivalence) between a conformal field theory in four dimensions with a non-abelian gauge group of degree $N$ and a ten-dimensional string theory on a curved space-time background. More specifically, the most studied version of the conjecture considers $\mathscr{N}=4$ super Yang-Mills theory with gauge group $S U(N)$ involving two limits: first, one makes $N \rightarrow \infty$ keeping $\lambda \equiv g^{2} N$ constant, ${ }^{1}$ and then, one sends $\lambda \rightarrow \infty$. According to the conjecture, this theory should be equivalent to Type IIB supergravity (i.e., the low-energy limit of Type IIB string theory) on a $A d S_{5} \times S^{5}$ background.

In spite of being a conformal field theory and of its high degree of supersymmetry, $\mathscr{N}=4$ SYM constitues an excellent test ground for phenomena which are of crucial importance in physical non-abelian gauge theories, in particular QCD. ${ }^{2}$ For instance, the analysis of the shear viscosity in this theory led to conjecture a lower bound for the quotient shear viscosity/entropy density in a class of theories with gravity duals [2]. ${ }^{3}$ And experimentally it turned out that the state of matter created in heavy-ion collisions (the Quark-Gluon Plasma) at RHIC and the LHC has near the cross over phase transition a value for this quotient very close to the conjectured bound [4]. Other observables which are currently studied intensively are correlation functions involving gauge-invariant operators and their relationship with Wilson loops, scattering amplitudes, or the study of the quarkantiquark potential by means of powerful techniques available due to the integrability of planar $\mathscr{N}=4$ SYM theory [5]. The initial conjecture has evolved significantly since it was introduced fifteen years ago: deformations of the field theory have been considered in order to build theories which resemble more QCD and other physical systems, so one can study phenomena of not only theoretical but also phenomenological interest such as confinement, dynamical chiral symmetry breaking, "hadrons", transport, jet quenching, etc. There is also an intense effort to apply the conjecture to theories in lower dimensions, with the aim of tackling condensed-matter problems (see e.g. [6]).

In this work we are interested in studying processes, such as synchrotron radiation or photon splitting, in magnetic fields of arbitrary intensity using the AdS/CFT duality. These phenomena are well known in the context of QED, but there they are usually analyzed at weak coupling using perturbative techniques. We shall argue that when the coupling constant is not small or when the magnetic field is intense enough, higher-order effects such as dimensional reduction start playing an important role and change the qualitative picture of these processes with respect to the leadingorder perturbative result. The physics of intense fields is reaching new frontiers nowadays. On one hand, non-central heavy-ion collisions at RHIC and LHC can generate magnetic and electric

\footnotetext{
${ }^{1} g$ is the gauge coupling, and $\lambda$ is called the 't Hooft coupling.

${ }^{2}$ Some have referred to $\mathscr{N}=4$ SYM theory as "the Lithium atom of the 21 st century" (cf. the plenary talk by Prof. Lance Dixon at this conference).

${ }^{3}$ So far, the AdS/CFT conjecture is the only reliable framework for computing transport coefficients of non-abelian gauge theories at strong coupling. Lattice calculations of these observables still suffer from large statistical errors, and the extension to finite densities is even more complicated due to the sign problem (for an introduction see [3]).
} 
fields of order $|e B| \sim|e E| \sim 10^{4} \mathrm{MeV}^{2} \sim 10^{13} \mathrm{~T}$, or even higher. These are fields $10^{5}$ larger than the critical electron field $\left|e B_{\mathrm{c}}\right| \sim\left|e E_{\mathrm{c}}\right| \sim m_{e}^{2} \sim 0.25 \mathrm{MeV}^{2}$, and therefore extremely intense. Under these strong fields, exotic phenomena such pair production out of the vacuum (Schwinger effect) can occur, and there is a chance of observing it (maybe indirectly) for the first time in an experiment. On the other hand, the experiments involving high-intensity lasers are close to reach the critical field threshold. This will allow in a more controlled way to study these non-linear QED effects and others such light-by-light scattering or photon splitting. For a broad exposition of the physics of strong fields see for instance [7].

Hence, analyzing these phenomena in a controlled theoretical framework which allows to consider electric and magnetic field of arbitrary intensity is of great value in order to get further insight into the physics of intense fields. In what follows we focus on synchrotron radiation as one of these possible phenomena.

\section{Synchrotron radiation}

It is a well known result of classical electrodynamics that charged particles radiate when accelerated by an electric or magnetic field. In the relativistic case, the power radiated is given by the Liénard formula [8]:

$$
P=\frac{2}{3} q^{2} \gamma^{6}\left[\dot{\boldsymbol{\beta}}^{2}-(\boldsymbol{\beta} \times \dot{\boldsymbol{\beta}})^{2}\right]
$$

where $q$ is the charge of the particle, $\boldsymbol{\beta}$ is its velocity, and $\gamma$ is the Lorentz factor. The angular distribution of the radiation depends on the type of motion the particle has. For instance, in the case of instantaneous rectilinear and circular motions, the distributions are respectively:

$$
\frac{\mathrm{d} P_{\text {rect. }}}{\mathrm{d} \Omega}=\frac{q^{2} \dot{\beta}^{2}}{4 \pi} \frac{\sin ^{2} \theta}{(1-\beta \cos \theta)^{5}} ; \quad \frac{\mathrm{d} P_{\text {circ. }}}{\mathrm{d} \Omega}=\frac{q^{2}}{4 \pi} \frac{\dot{\beta}^{2}}{(1-\beta \cos \theta)^{3}}\left[1-\frac{\sin ^{2} \theta \cos ^{2} \phi}{\gamma^{2}(1-\beta \cos \theta)^{2}}\right]
$$

where $\boldsymbol{\beta}$ is assumed to point in the $z$-direction, $\theta$ denotes the polar angle of a vector $\boldsymbol{n}$ pointing in the direction of observation, and the acceleration in the circular case is assumed to point in the $x$-direction, with $\phi$ the azimuthal angle of $\boldsymbol{n}$. In the non-relativistic case, a particle in rectilinear motion emits most of its radiation in the directions orthogonal to its velocity, whereas the particle in circular motion emits most of its radiation in the direction for which the quantity $\sin ^{2} \theta \cos ^{2} \phi$ is minimum. The common feature of the pattern of radiation in both types of motion is that when the velocity approaches the speed of light, the radiation gets collimated in a cone around the direction of motion win an opening angle $\sim 1 / \gamma$. In the ultra-relativistic regime, the power of radiation emitted in circular motion is much larger than in the linear case: $P_{\text {circ. }} \sim \gamma^{2} P_{\text {rect. }}$. This is essentially the reason why circular accelerators have much larger energy losses in the beam than linear accelerators.

Synchrotron radiation has also been studied at strong coupling in the context of the AdS/CFT correspondence, starting with the work [9]. There, the author found that the power radiated by an accelerated test quark in $\mathscr{N}=4$ SYM theory at strong coupling has the same simple expression as (2.1) except for the substitution $\frac{2}{3} q^{2} \leftrightarrow \sqrt{\lambda} / 2 \pi$. This result has been confirmed by later works, 
[10-13]. ${ }^{4}$ In particular, in [10], the authors studied the radiation emitted from a trailing string, which corresponds to a quark in the fundamental representation, moving in a circular trajectory. They obtained two main, and in principle unexpected, results: (i) the radiation is beamed in the direction of the velocity of the quark in a cone with opening angle $\sim 1 / \gamma$ and the power emitted fullfils the Lienard formula. (ii) The radiation propagates without broadening in pulses of width $\Delta \sim 1 / \gamma^{3}$ (i.e., the radiation does not isotropize). We shall argue that these results are a consequence of forcing the particle to follow a classical trajectory, the use of the quenched approximation, and the property that, unlike QED, the theory is conformal.

The fact that a quantum gauge field theory at strong coupling gives formally the same result as in classical electrodynamics is certainly surprising. Specially taking into account that a leadingorder calculation in SQED (see Figure 1) already provides a result which differs from the classical one. For instance, for a charged scalar field coupled to an external magnetic field, the first quantum correction to the classical result for the power radiated is negative [14]:

$$
P=P_{\mathrm{cl}}\left[1-3.97 \frac{|e| B E}{m^{3}}+\mathscr{O}\left(\xi^{2}\right)\right], \quad \xi \equiv \frac{3|e| B E}{2 m^{3}},
$$

where $E$ is the energy of the particle, and $m$ its mass.

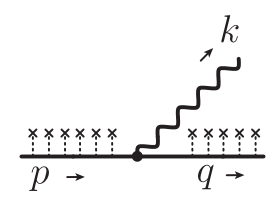

Figure 1: A charged scalar particle of momentum $p$ interacts with an external magnetic field and emits a photon of momentum $k$.

Quantum effects enter in two ways:

1. Quantization of the motion of the charged particle: characterized by the quotient $\Delta E / E$, where $\Delta E$ is the separation between two Landau levels in the spectrum of the particle, and $E$ is the energy of the particle. The classical motion corresponds to $\Delta E / E \rightarrow 0$.

2. Quantum recoil: characterized by the quotient $k / E$, where $k$ is the energy of the emitted photon. The classical limit corresponds to $k / E \rightarrow 0$. However, as we increase $E$, the average momentum of the emitted photon also increases as $\bar{k} \sim|e| B E^{2} / m^{3}$ [14]. Therefore, this is a quantum effect which becomes important at high energies.

But there is an additional quantum effect which has not been considered so far when analyzing synchrotron radiation, and it is the phenomenon of dimensional reduction in a strong magnetic field. Consider for instance the contribution from the lowest Landau level to the fermion propagator in a magnetic field $[15,16]$ :

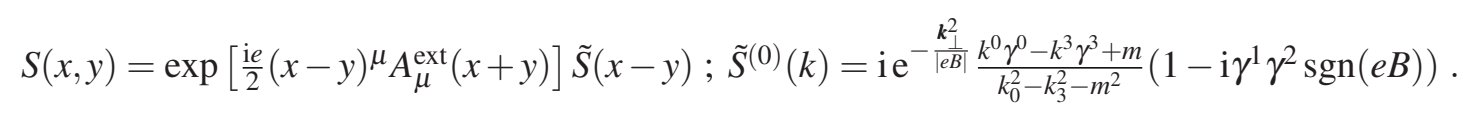

\footnotetext{
${ }^{4}$ In [12], however, they also considered an acceleration caused by means of an external field that couples only at tree level to the quark (there are no quark loops, i.e., they consider the quenched approximation). In this case they do find a deviation form the Lienard formula.
} 
Then, it is not difficult to realize that, for a very intense magnetic field, the lowest Landau level dominates fermionic loops, giving a contribution for instance to the photon propagator shown in Figure 2 of order $\sim|e| B$. Hence, the kinematic constraints imposed over the final photons by the

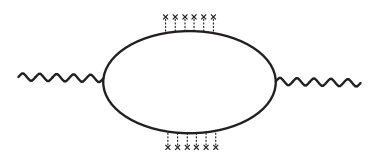

Figure 2: Correction to the photon propagator due to a fermion loop which interacts with the external magnetic field. This contribution becomes much larger than the leading-order propagator if the magnetic field is strong-enough.

fermions in the loops with momenta aligned with the magnetic fields imply that, for very strong magnetic fields, most of the radiation will be emitted in the direction of the magnetic field. This pattern is certainly very different from the classical result, and it is purely quantum mechanical (it is crucial the interaction of the external field with the fermion loops).

\section{Magnetic brane and radiation processes at strong coupling}

In order to couple the $\mathscr{N}=4 \mathrm{SYM}$ theory to an external (non-dynamical) magnetic field, we consider the deformed geometry obtained by solving the Einstein-Maxwell equations in $4+1$ dimensions [17]. Regarding synchrotron radiation, when trying to make a fair comparison between QED and $\mathscr{N}=4$ SYM theory at strong coupling, we face two main problems: (i) at strong coupling, it is no longer possible to distinguish between the original charged particles and charged particles which are part of the radiation (see Fig. 3); (ii) the AdS/CFT conjecture only allows to compute amplitudes involving gauge-invariant composite operators (e.g., it is not possible to obtain the quark or gluon propagator in this framework). In order to address the first issue, we can couple

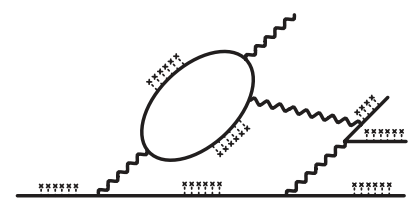

Figure 3: At strong coupling, it is not possible to distinguish radiation from the original particle.

$\mathscr{N}=4 \mathrm{SYM}$ theory to $N_{f} \ll N$ (quenched) flavors of fundamental matter. These flavors are neutral under the external magnetic field but they still experience acceleration by means of the coupling to virtual particles. The second issue can be overcome by considering meson operators containing these flavor fields. These states correspond to quasiparticles with small widths.

The analysis of the pattern of radiation is then carried out in the same spirit as in [18]. Semianalytical results can be obtained for weak and very strong magnetic fields, but it is technically involved. Work is in progress and the final result will be published soon [19]. 


\section{Acknowledgments}

This work is supported by an Alexander von Humboldt fellowship. Partial financial support from the Spanish Research projects FIS2008-01323 and FPA2011-27853-C02-02 is also acknowledged.

\section{References}

[1] J.M. Maldacena, The large N limit of superconformal field theories and supergravity, Adv. Theor. Math. Phys. 2 (1998) 231 [hep-th/9711200].

[2] P. Kovtun, D.T. Son and A.O. Starinets, Viscosity in strongly interacting quantum field theories from black hole physics, Phys. Rev. Lett. 94 (2005) 111601.

[3] G. Aarts, Two complex problems on the lattice: Transport coefficients and finite chemical potential, Nucl. Phys. A 820 (2009) 57C.

[4] P. Romatschke and U. Romatschke, Viscosity information from relativistic nuclear collisions: How perfect is the fluid observed at RHIC?, Phys. Rev. Lett. 99 (2007) 172301.

[5] N. Beisert et al., Review of AdS/CFT integrability: An overview, Lett. Math. Phys. 99 (2012) 3.

[6] S.A. Hartnoll, Lectures on holographic methods for condensed matter physics, Class. Quant. Grav. 26 (2009) 224002.

[7] K. Itakura, et al. (eds), Proceedings of International Conference on Physics in Intense Fields (PIF2010), 24-26 November 2010, KEK, Tsukuba, Japan [http://ccdb5fs.kek.jp/tiff/2010/1025/1025013.pdf].

[8] J.D. Jackson, Classical electrodynamics, John Wiley \& Sons Inc., Hoboken 1999.

[9] A. Mikhailov, Nonlinear waves in AdS/CFT correspondence, hep-th/0305196.

[10] C. Athanasiou, P. M. Chesler, H. Liu, D. Nickel and K. Rajagopal, Synchrotron radiation in strongly coupled conformal field theories, Phys. Rev. D 81 (2010) 126001 [Erratum-ibid. 84 (2011) 069901] [arXiv: 1001.3880 [hep-th]].

[11] R. Baier, On radiation by a heavy quark in N = 4 SYM, Adv. High Energy Phys. 2012 (2012) 592854 [arXiv:1107.4250 [hep-th]].

[12] M. Chernicoff, J.A. Garcia, A. Guijosa and J.F. Pedraza, Holographic lessons for quark dynamics, J. Phys. G 39 (2012) 054002 [arXiv: 1111.0872 [hep-th] ].

[13] Y. Hatta, E. Iancu, A.H. Mueller and D.N. Triantafyllopoulos, Radiation by a heavy quark in $N=4$ SYM at strong coupling, Nucl. Phys. B 850 (2011) 31 [arXiv: 1102.0232 [hep-th] ].

[14] A.A. Sokolov and I.M. Ternov, Synchrotron radiation, Pergamon Press Ltd., Oxford 1968.

[15] J. S. Schwinger, On gauge invariance and vacuum polarization, Phys.Rev. 82 (1951) 664.

[16] V.P. Gusynin, V.A. Miransky and I.A. Shovkovy, Dimensional reduction and catalysis of dynamical symmetry breaking by a magnetic field, Nucl. Phys. B 462 (1996) 249 [hep-ph / 9509320 ].

[17] E. D'Hoker and P. Kraus, Magnetic brane solutions in AdS, JHEP 0910 (2009) 088 [arXiv:0908.3875 [hep-th]].

[18] D.M. Hofman and J. Maldacena, Conformal collider physics: Energy and charge correlations, JHEP 0805 (2008) 012 [arXiv:0803.1467 [hep-th] ].

[19] D. Fernandez-Fraile, in preparation, (2013). 